\title{
Hydrodynamic and electrokinetic effects on the dynamics of charged colloids and macromolecules
}

\author{
Adolfo J. Banchio \\ CONICET and FaMAF, Universidad Nacional de Córdoba, Ciudad Universitaria, \\ X5000HUA Córdoba, Argentina

\section{Mathieu G. McPhie and Gerhard Nägele} \\ Institut für Festkörperforschung, Teilinstitut Weiche Materie, Forschungszentrum \\ Jülich, D-52425 Jülich, Germany \\ E-mail: g.naegele@fz-juelich.de
}

\begin{abstract}
We explore dynamic processes in suspensions of charge-stabilized colloidal particles and biological macromolecules. Various short-time transport properties including diffusion functions and the high-frequency viscosity, have been calculated by means of a recently developed accelerated Stokesian Dynamics simulation tool. This has allowed us to study dense suspensions with many-body hydrodynamic interactions. The results of this study are used to explore the validity of generalized StokesEinstein relations, and the possibility of measuring self-diffusion at a finite scattering wave number. The influence of the electrolyte ion dynamics on the colloidal longtime self-diffusion in non-dilute suspensions of small colloids is determined using a many-component mode-coupling theory that accounts for the inter-ionic hydrodynamic interactions. We investigate the influence of the asymmetry in the electrolyte ion charges and mobilities on the friction experienced by the charged colloids. It is shown that these asymmetries affect the electrolyte friction significantly, and that the electrolyte friction contribution to self-diffusion decreases with increasing colloid concentration.
\end{abstract}




\section{Introduction}

The dynamics of liquid dispersions of charged colloidal particles is of fundamental interest in soft matter science, surface chemistry and food industry. The scope for these systems has been broadened even further through the increasing importance of biophysical research dealing with charged biomolecules like proteins and DNA. Many of the theoretical methods and computer simulation techniques that have been developed in colloid physics are directly applicable to biological molecules and cells.

In exploring the dynamics of charge-stabilized colloids theoretically, a complicated interplay of inter-particle forces has to be considered. The colloidal macroions and the neutralizing small counterions and coions (microions) interact with each other through electrosteric and hydrodynamic interactions. The latter type of forces, transmitted quasi-instantaneously on colloidal time scales by the intervening solvent molecules, is very long-ranged and in general of many-body nature. This causes challenging problems in the theory and computer simulation studies of the colloid dynamics.

Two major routes are followed in theoretical studies on the dynamics of charged colloids. In the first, a charge-stabilized suspension is modeled as an effective onecomponent fluid of microion-dressed colloidal macroions, interacting at non-overlap distances by a spherically symmetric screened Coulomb potential. In this so-called one-component macroion fluid model (OMF), the microions enter into the description only through an effective screening parameter and an effective colloid charge. The OMF model thus disregards the non-instantaneous dynamic response of the microionic atmosphere to the colloid motion. In the second part of this paper, where a more refined many-component model is used, we will show that this is a good approximation for colloidal particles much larger than the electrolyte ions. The simplicity of the onecomponent model allows for a quantitative consideration of the strong colloid-colloid hydrodynamic interactions (HI), and for the study of their effects in dense chargestabilized suspensions.

The present paper consists of two parts. In the first part (section 2), we present a simulation study of short-dynamic properties of dense suspensions on the basis of the OMF model. Using a recently developed accelerated Stokesian dynamics (ASD) simulation method for Brownian spheres [1], we have calculated and analyzed a variety of short-time dynamic properties such as translational and rotational self-diffusion coefficients, wave-number dependent diffusion functions, and the high-frequency-limiting viscosity. The ASD simulation method accounts both for many-body HI and lubrication effects. The results of our simulation study are used to scrutinize the validity of shorttime generalized Stokes-Einstein (GSE) relations linking the high-frequency viscosity to diffusion properties, and to explore the possibility to measure the self-diffusion coefficient in a dynamic scattering experiment at a specifically selected wave number.

The second route to describe the dynamics of charged colloidal particles relies on a more refined multi-component description, the so-called dynamic Primitive Model, in which the colloidal particles, surface-released counterions and electrolyte ions are 
treated alike as charged hard spheres interacting via excluded volume, electrostatic and hydrodynamic forces. Just like in the OMF model, the solvent is treated as a structureless hydrodynamic continuum, and the time evolution of all ionic species is determined by a many-component Smoluchowski equation. While the multi-component model allows, in principle, to consider electrolyte ion kinetic effects on the colloid diffusion and rheology arising from their finite response times, it is far more costly to deal with than the OMF model, particularly in computer simulations. Moreover, additional approximations must be introduced in theoretical calculations.

Section 3 includes the second part of the paper, where we use the dynamic Primitive Model as the starting point to study the dynamic influence of salt ions on the long-time self-diffusion of small colloidal particles or biomolecules in a non-dilute suspension. Our calculation of the colloidal long-time self-diffusion coefficient in this model is based on a simplified many-component mode-coupling scheme which requires the inter-ionic static pair correlation functions as the only input. This scheme is a marked improvement on previous methods, since it includes the far-field HI between all ionic species and allows the study of electrokinetic effects in colloidal suspension at non-zero colloid concentrations [2]. We explore in particular the influence of asymmetry in the electrolyte ion charges and mobilities on the long-time self-friction experienced by the charged colloids. It is shown that these asymmetries have a significant effect on the electrolyte friction.

\section{Colloidal Short-time Dynamics}

\subsection{One-component macroion model}

Our computer simulation study of short-time dynamic properties in dense suspensions of charge-stabilized colloidal spheres and the analytic theory calculations discussed in this paper, are based on the one-component macroion fluid model. The colloidal spheres with their clouds of neutralizing microions are described in this simplifying model as uniformly charged hard spheres interacting by the effective pair potential of DerjaguinLandau-Verwey-Overbeek (DLVO) type [3]

$$
\frac{u(r)}{k_{B} T}=L_{B} Z^{2}\left(\frac{e^{\kappa a}}{1+\kappa a}\right)^{2} \frac{e^{-\kappa r}}{r}, \quad r>2 a .
$$

Here, the electrostatic screening parameter, $\kappa$, is given by

$$
\kappa^{2}=\frac{4 \pi L_{B}\left[n|Z|+2 n_{s}\right]}{1-\phi}=\kappa_{c i}^{2}+\kappa_{s}^{2},
$$

where $n$ is the colloid number density, $n_{s}$ is the number density of added 1-1 electrolyte, and $\phi=(4 \pi / 3) n a^{3}$ is the colloid volume fraction of spheres with radius $a$. Furthermore, $Z$ is the charge on a colloid sphere in units of the elementary charge $e$, and $L_{B}=$ $e^{2} /\left(\epsilon k_{B} T\right)$ is the Bjerrum length of the suspending fluid of dielectric constant $\epsilon$ at temperature $T$. The solvent is characterized solely by its dielectric constant $\epsilon$ and the shear viscosity $\eta_{0}$. The square of the screening parameter has a contribution, $\kappa_{c i}^{2}$, 
due to surface-released counter-ions, which are assumed here to be monovalent, and a contribution, $\kappa_{s}^{2}$, arising from the added electrolyte. The factor $1 /(1-\phi)$ corrects for the free volume accessible to the microions [4].

For strongly charged spheres with $L_{B}|Z| / a>1, Z$ should be interpreted as an effective charge number smaller than the bare one, owing to the quasi-condensation of counterions close to the colloid surfaces. Likewise, a renormalized value for $\kappa$ should be used. Several schemes have been developed to relate the effective $Z$ and $\kappa$ to the bare ones $[5,6,7,8]$. The outcome of these schemes depends on the approximation made for the free energy functional, and on additional simplifications used such as the spherical cell or macroion jellium models.

We do not embark here on the ongoing discussion on how the effective charge and screening parameters are related to their bare counterparts, and on how one can generalize the charge-renormalization concept to dynamic phenomena. We only mention here that an interesting relation between charge-renormalization and long-time selfdiffusion of DNA fragments is discussed in a forthcoming article [9].

In the present simulation study, the OMF model is used as a well-established model that captures essential features of charge-stabilized suspensions. It allows us to explore general trends in the short-time dynamics of charged dispersions by an affordable amount of computation time. Our focus will be on hydrodynamic interaction effects.

\subsection{Short-time dynamic properties}

In colloid dynamics, one distinguishes the short-time regime, $\tau_{B} \ll t \ll \tau_{I}$, from the long-time regime characterized by $t \gg \tau_{I}[10,11]$. The interaction time $\tau_{I}$ can be estimated by $a^{2} / D_{0}$, where $D_{0}=k_{B} T /\left(6 \pi \eta_{0} a\right)$ is the translational diffusion coefficient of a single sphere suspended in a fluid of viscosity $\eta_{0}$. It characterizes the time span required for direct particle interactions to become influential. The relaxation time of the colloid momenta is denoted by $\tau_{B}$. At long times $t \gg \tau_{I}$, the motion of particles is diffusive due to the incessant bombardment by solvent molecules and interactions with other surrounding particles. Within the colloidal short-time regime addressed in the present section, the configuration of particles has changed so little that the slowing influence of the direct interactions is not yet operative. However, the short-time dynamics is influenced by the solvent mediated hydrodynamic interactions which, in the case of colloids, act quasi-instantaneously. In unconfined suspensions of mobile particles, the HI are long-range and, in general, non-pairwise additive. The HI strongly affect the diffusion and rheology in dense suspensions, and they can give rise to unexpected dynamic effects.

Long-time diffusion properties are always smaller than the corresponding short-time

ones, since dynamic caging by neighboring particles becomes operative at longer times only, where it has a slowing influence on the particle diffusion. Short-time properties, on the other hand, are not subject to dynamic caging. Therefore, they can be expressed in terms of equilibrium averages, in which direct interactions appear only indirectly 
through their influence on the equilibrium microstructure.

Short-time dynamics properties can be probed experimentally by a variety of scattering techniques. For example photon correlation spectroscopy probes the dynamic structure factor, $S(q, t)$ as a function of scattering wave number $q$ and correlation time $t$. For short correlation times where $\tau_{B} \ll t \ll \tau_{I}, S(q, t)$ decays exponentially according to $[10,11]$

$$
\frac{S(q, t)}{S(q)} \approx \exp \left[-q^{2} D(q) t\right]
$$

with a $q$-dependent short-time diffusion function, $D(q)$, that can be expressed by the ratio

$$
D(q)=D_{0} \frac{H(q)}{S(q)},
$$

of the hydrodynamic function $H(q)$, and the static structure factor $S(q)=S(q, t=0)$. The microscopic expression for $H(q)$ is given by [11]

$$
H(q)=\left\langle\frac{k_{B} T}{N D_{0}} \sum_{l, j=1}^{N} \hat{\mathbf{q}} \cdot \mu\left(\mathbf{r}^{N}\right)_{l j} \cdot \hat{\mathbf{q}} \exp \left\{i \mathbf{q} \cdot\left[\mathbf{r}_{l}-\mathbf{r}_{j}\right]\right\}\right\rangle,
$$

where $N$ is the number of spheres in the scattering volume, $\mathbf{r}_{j}(t)$ is the position vector of sphere $j$ at time $t, \hat{\mathbf{q}}$ is the unit vector in the direction of the scattering wavevector $\mathbf{q}$, and the brackets represent an equilibrium ensemble average. Furthermore, $\mu\left(\mathbf{r}^{N}\right)_{l j}$ is the translational hydrodynamic mobility tensor that relates the hydrodynamic force on a sphere $j$ to the resulting velocity change of sphere $l$. It depends in general on the configuration, $\mathbf{r}^{N}$, of all $N$ particles which makes an analytic calculation of $H(q)$ intractable, unless approximations can be introduced.

The function $H(q)$ contains the influence of the HI on the short-time diffusion. It is the sum of a $q$-independent self-part, and a $q$-dependent distinct-part, $H(q)=D_{s} / D_{0}+$ $H_{d}(q)$, where $D_{s}$ is the short-time translational self-diffusion coefficient proportional to the initial slope of the particle mean squared displacement. At wavenumbers large compared to the position, $q_{m}$, of the principal peak in $S(q), H(q)$ becomes equal to $D_{s} / D_{0}$. In the opposite limit of very small $q, H(q)$ is equal to the short-time sedimentation velocity, $U_{s}$, of a slowly settling homogeneous suspension taken relative to the sedimentation velocity, $U_{0}$, at infinite dilution. Without $\mathrm{HI}, H(q)$ is identically equal to one so that any $q$-dependence signals the influence of HI.

For $q a \ll 1, D(q)$ reduces to the short-time collective diffusion coefficient, $D_{c}$, which for strongly charged particles at low salinity is substantially larger than the free diffusion coefficient $D_{0}$. In the large- $q$ limit, $D\left(q \gg q_{m}\right) \approx D_{s}$. Note that $D_{s}$ is smaller than $D_{0}$ at non-zero colloid concentrations.

Additional short-time properties explored in our simulation study are the shorttime rotational self-diffusion coefficient, $D_{r}$, which is the rotational analog of $D_{s}$, and the high-frequency-limiting suspension viscosity $\eta_{\infty}$. The short-time viscosity $\eta_{\infty}$ is measured in low-amplitude shear oscillation rheometers at frequencies $\omega \tau_{I} \gg 1$ high enough so that the equilibrium microstructure is unaffected by the imposed shear flow. 
Very recently [12], we have reported on a comprehensive simulation study on the short-time dynamics of charged colloidal spheres described in the OCM model. In the following, an extension of this study will be presented with additional new results.

\subsection{Simulation and theory}

We have calculated a variety of short-time dynamic properties using the accelerated Stokesian dynamics (ASD) simulation code developed by Banchio and Brady [1]. We have extended this code to charged spheres described by the OMF pair potential. The ASD code allows us to simulate short-time properties for a larger number of colloidal spheres, typically up to one thousand placed in a periodically replicated simulation box, which gives improved statistics. The details of the ASD simulation method, which accounts for many-body HI and lubrication effects, have been given in Ref. [1]. To speed up the computation of short-time quantities such as $H(q)$, a set of equilibrium configurations has been generated using a Monte-Carlo simulation code in the case of charge-stabilized spheres, and a Molecular Dynamics simulation code for neutral hard spheres. Finite-size effects arising from the periodic boundary conditions have been corrected for using a finite-size scaling procedure introduced by Ladd and coworkers in earlier Lattice-Boltzmann simulations of colloidal hard spheres [13, 14, 15].

The only analytic method available to date that allows the prediction of $H(q), D_{s}$ and $\eta_{\infty}$ of dense suspensions of neutral and charge-stabilized spheres is the (zeroth order) renormalized density fluctuation expansion method of Beenakker and Mazur $[16,17]$. This so-called $\delta \gamma$ method is based on a partial resummation of many-body HI contributions, and it invokes truncated hydrodynamic mobility tensors. To leading order in the renormalized density fluctuations, the hydrodynamic function in this scheme is obtained from $[16,18,19,20]$

$H_{d}(q)=\frac{3}{2 \pi} \int_{0}^{\infty} d(a k)\left(\frac{\sin (a k)}{a k}\right)^{2}\left[1+\phi S_{\gamma_{0}}(a k)\right]^{-1} \times \int_{-1}^{1} d x\left(1-x^{2}\right)[S(|\mathbf{q}-\mathbf{k}|)-1]$

and

$$
\frac{D_{s}(\phi)}{D_{0}}=\frac{2}{\pi} \int_{0}^{\infty} d t\left(\frac{\sin t}{t}\right)^{2}\left[1+\phi S_{\gamma_{0}}(t)\right]^{-1} .
$$

Here, $x$ is the cosine of the angle extended by the wave vectors $\mathbf{q}$ and $\mathbf{k}$, and $S_{\gamma_{0}}(t)$ is a known function independent of the particle correlations and given in $[16,18]$. The only input is the static structure factor $S(q)$, which we have calculated here using the rescaled mean-spherical (RMSA) integral equation scheme. Actually, $S(q)$ is input to the distinct part of $H(q)$ only, whereas the expression for $D_{s}$ is independent of $S(q)$. In the application of the zeroth-order $\delta \gamma$ scheme to charge-stabilized spheres, $D_{s}$ is thus more roughly approximated by the value for neutral hard spheres at the same volume density $\phi$, independent of the particle charge and the screening parameter. To account for the actual pair correlations in calculating $D_{s}$ requires to go one step further in the fluctuating density expansion, which severely complicates the scheme. However, from comparing the $\delta \gamma$ scheme predictions with our ASD simulation results and a large 


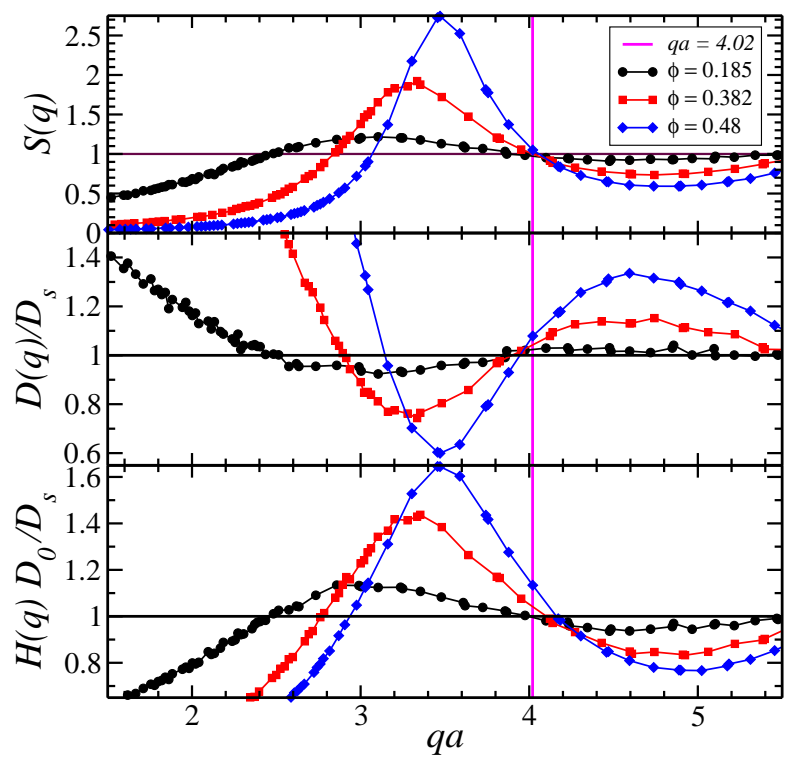

Figure 1. Static structure factor $S(q)$, short-time diffusion function $D(q)$ and hydrodynamic function $H(q)$ of hard-sphere suspensions at volume fractions $\phi$ as indicated. The dynamic properties are scaled by the respective short-time self-diffusion coefficient $D_{s}(\phi)$. To a good approximation, there is an isobestic point at $q_{s} a \approx 4.02$, where $S(q)$ and $H(q) \times\left(D_{0} / D_{s}\right)$ are equal to 1 (see vertical line). Our ASD simulation data are denoted by symbols.

collection of experimental data on charge-stabilized systems, we have found that $H_{d}(q)$ is well described by equation (6). This allows the improvement of the $\delta \gamma$ scheme by replacing the hard-sphere $\delta \gamma$ prediction for $D_{s}$ by the accurate simulation value which is a less time-consuming method than the full ASD simulation of $H(q)$. The hybrid method amounts to an upward shift of the $\delta \gamma-H(q)$ by a small to moderately large value, since the $D_{s}$ of charged spheres is larger than that of neutral ones [11,21]. Even without the correction for $D_{s}$, the $\delta \gamma$ scheme is useful in detecting trends in the behavior of $H(q)$.

In the following, we discuss our ASD simulation results for a collection of shorttime dynamic properties. The system parameters used are $\epsilon=10, T=298.15 \mathrm{~K}$, sphere radius $a=100 \mathrm{~nm}, n_{s}=0$ in general, and a colloid charge number $Z=100$ corresponding to $L_{B}|Z| / a=5.62$. It will be noted in the captions when different parameters have been used. The present parameters are representative of suspensions of strongly charged colloidal spheres.

\subsection{Hydrodynamic and short-time diffusion functions}

We start by discussing the general behavior of the hydrodynamic function $H(q)$, the short-time self-diffusion function $D(q)$, normalized by the short-time self-diffusion coefficient $D_{s}(\phi)$, and the static structure factor $S(q)$. The ASD simulation results for neutral hard spheres, and charge-stabilized spheres at zero added salt, are depicted in figures 1 and 2, respectively, for volume fractions $\phi$ as indicated. It should be noted that 


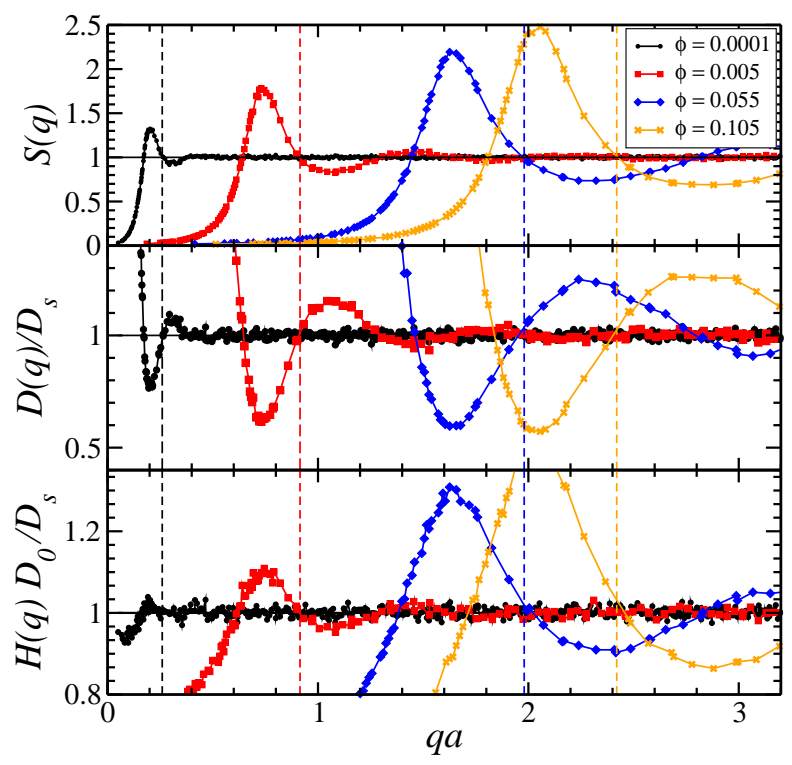

Figure 2. Same as in figure 1 but for deionized suspensions of charged spheres at volume fractions as indicated. In the present systems, there are no isobestic points. The vertical dashed lines mark the wave numbers, $q_{s}$, where $S\left(q_{s}\right)=1$.

$H(q) \rightarrow D_{s} / D_{0}$ and $D(q) \rightarrow D_{s}$ for large values of $q$. The undulations of $H(q)$ and $D(q)$ in $q$ are in phase with those of $S(q)$. In particular, the maximum (minimum) of $H(q)$ $(D(q))$ is located right at the peak position, $q_{m}$, of $S(q)$. For hard spheres, where nearfield $\mathrm{HI}$ is dominant, $H\left(q_{m}\right)$ decreases linearly in $\phi$, according to $H\left(q_{m}\right)=1-1.35 \phi$, for concentrations extending up to the freezing transition [12]. In contrast to this, in saltfree suspensions of strongly charged spheres, $H\left(q_{m}\right)$ increases sublinearly in $\phi$ at small concentrations, characterized by an exponent of about 0.4 . This slow increase arises from the dominance of the far-field HI part in systems where the particles stay apart from each other because of long-range electrostatic repulsions. With increasing $\phi$, however, the slowing influence of near-field HI comes into play so that $H\left(q_{m}\right)$ passes through a maximum [23]. In the systems of figure 2 , this maximum is reached at $\phi \approx 0.06$.

The slowest decay of density fluctuations of wavelength $2 \pi / q$, as quantified by the minimum $D\left(q_{m}\right)$ in $D(q)$, is found for the wavelength $2 \pi / q_{m}$ that characterizes the size of a next-neighbor cage. This cage stiffens with increasing $\phi$. Therefore, the cage diffusion coefficient $D\left(q_{m}\right)$ decreases with increasing concentration by a rate that increases with particle charge (see figure 3). The strong sensitivity of $D\left(q_{m}\right)$ on interaction parameters such as the particle charge is due to its explicit dependence on the inverse of $S\left(q_{m}\right)$. In charged-sphere suspensions at low salinity, $S\left(q_{m} ; \phi\right)$ increases initially very strongly with $\phi$, since only few counterions are available to screen the electrostatic repulsion. This, in turn, causes the sharp drop in $D\left(q_{m}\right)$ at very small $\phi$, as can be seen in the figure .

When an increasing amount of salt is added, both $H\left(q_{m}\right)$ and $H\left(q \gg q_{m}\right)=D_{s} / D_{0}$ decline towards their hard-sphere limiting values. Opposite to this, the sedimentation coefficient, $H(0)$, increases with increasing salinity. This reflects the experimentally 


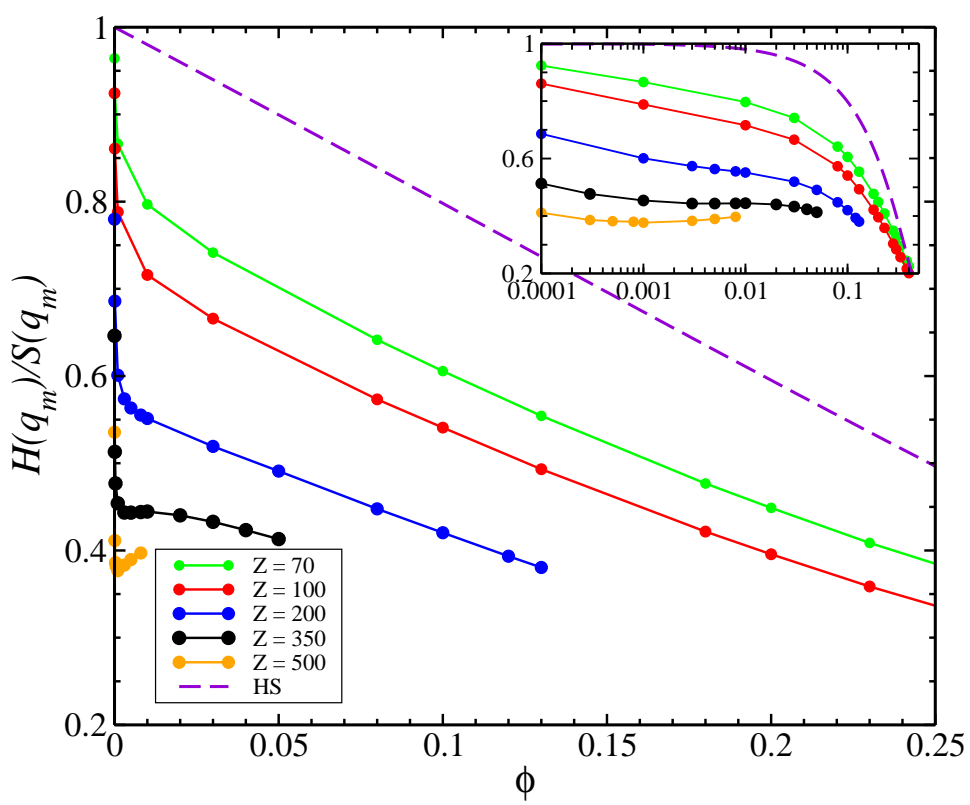

Figure 3. Cage diffusion coefficient of an aqueous deionized suspension versus volume fraction for various colloid charge numbers $Z$ as indicated. The system parameters are $a=25 \mathrm{~nm}, T=298 \mathrm{~K}, L_{B}=0.70 \mathrm{~nm}$ and $n_{s}=0$. The curves shown are obtained using the $\delta \gamma$ scheme with a RMSA structure factor input. Note the steep decline of $D\left(q_{m}\right)$ for very small $\phi$. The inset shows the same data plotted on a logarithmic volume fraction scale.

confirmed observation that charged particles at low salinity settle more slowly than uncharged ones $[12,24]$. Without exemption, both the ASD simulations and the analytic $\delta \gamma$ theory are in accordance with the general ordering relations,

$$
\begin{aligned}
& H^{C S}\left(q_{m}\right)>H^{H S}\left(q_{m}\right) \\
& D_{s}^{C S}>D_{s}^{H S} \\
& U_{s}^{C S}<U_{s}^{H S},
\end{aligned}
$$

where CS and HS are the labels, respectively, for charged and neutral hard spheres. For systems with added salt, the values of these short-time properties are located in between the zero-salt and infinite-salt (zero-charge) limits. Whereas the majority of experimentally available scattering data on charge-stabilized suspensions is in agreement with the ordering relations in equations (8) [22, 23], a violation of these relations has been purportedly observed in certain scattering experiments [25, 26].

Colloidal hard spheres have a common static and hydrodynamic length scale given by the particle radius $a$. This is the reason for the approximate existence of an isobestic wavenumber $q a \approx 4.02$ observed to the right of the main structure factor peak, where both $S(q)$ and $H(q) \times D_{0} / D_{s}$, and hence also $D(q) / D_{s}$, are equal to one, independent of the volume fraction (see figure 1 ). In fact, the wavenumber where $S(q)$ passes through 1 at $\phi=0.185$ is slightly smaller but approaches $4.02 / a$ with increasing $\phi$.

Unlike hard spheres, deionized suspensions of strongly charged spheres have at least two characteristic length scales, namely the geometric mean particle distance, $n^{-1 / 3}$, and 

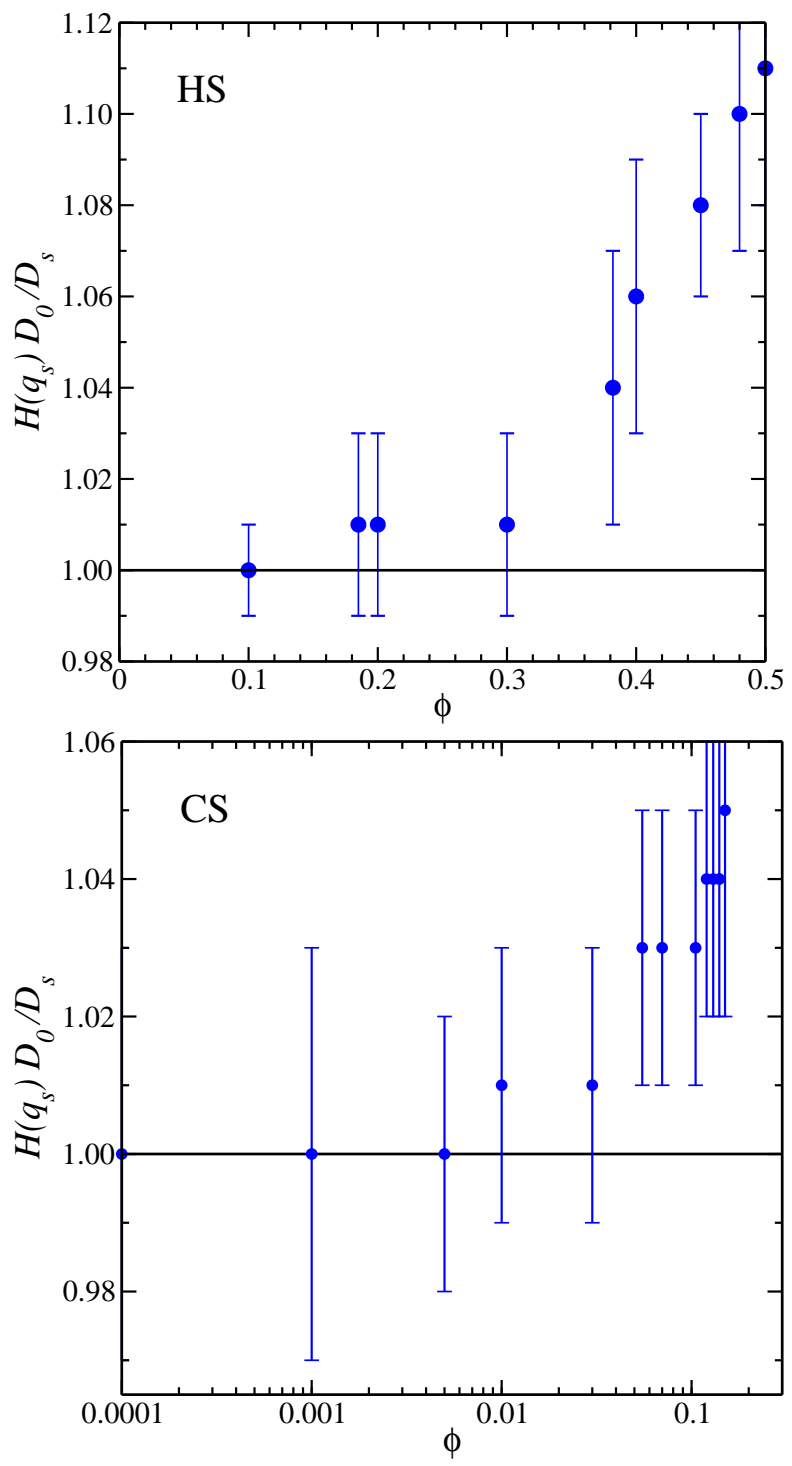

Figure 4. ASD simulation test on the validity of $D\left(q_{s}\right)=H\left(q_{s}\right) \approx D_{s}$ for neutral hard spheres (HS) and deionized charged-sphere suspensions (CS).

the hydrodynamic radius $a$. Consequently, in these systems there is no isobestic point for $S(q)$ and $H(q)$. The non-existence of a concentration-independent isobestic point in deionized suspensions is exemplified in figure 1.

It has been suggested by Pusey [27] that self-diffusion can be probed in a dynamic light scattering experiment performed at a wave number $q_{s}>q_{m}$, where $S\left(q_{s}\right)=1$. The assumption made here is that at such a wavenumber, where the distinct static structure factor, $S_{d}(q)=S(q)-1$, is zero, also its time-dependent generalization, $S_{d}\left(q_{s}, t\right)=S\left(q_{s}, t\right)-G\left(q_{s}, t\right)$, should remain small, in comparison to the self-intermediate scattering function, $G\left(q_{s}, t\right)$, that describes colloidal self-diffusion [10]. Note here that $G(q, 0)=1$ for all values of $q$. If this assumption holds true at least on an approximate level, then it is found indeed that $D_{s} \approx D_{0} H\left(q_{s}\right)=D\left(q_{s}\right)$. For all systems explored in our extensive ASD study, we find the difference between $D_{s} / D_{0}$ and $H\left(q_{s}\right)$ to be less 
than ten percent both for neutral and charged spheres (cf., figure 4). Thus dynamic light scattering experiments at such a point $q_{s}$ where $S\left(q_{s}\right)=1$, can be used to obtain a decent estimate for the value of $D_{s}$.

\subsection{High-frequency viscosity}

We proceed to discuss the concentration dependence of the high-shear limiting viscosity $\eta_{\infty}$, in the linear-response regime where shear-thinning remains absent. A lot of experimental and simulation data have been accumulated in the past for the $\eta_{\infty}$ of colloidal hard spheres [13, 28, 29]. Comparatively few investigations have been made on low-salt suspensions of charged particles [30, 31]. In figure 5, we display the ASD simulation results for two different deionized systems with system parameters as listed in the caption. These results for charged spheres should be compared with the ASD data for neutral hard spheres. As can be noticed, the viscosity difference between charged and neutral particles is quite small even at larger $\phi$. The fact that $\eta_{\infty}(C S)<\eta_{\infty}(H S)$ at all $\phi$ reflects the smaller hydrodynamic dissipation in charged-spheres systems due to the depletion of neighboring spheres near contact caused by the electrostatic repulsion. Figure 6 shows that the increase in viscosity due to the increased electrostatic screening triggered by the addition of salt is very weak. This weak ionic strength dependence of $\eta_{\infty}$, and its only modest increase with increasing volume fraction predicted by the ASD simulations, are features fully consistent with the experimental findings of Bergenholtz et al. [30].

Our ASD simulation data for hard spheres are well described by

$$
\frac{\eta_{\infty}}{\eta_{0}}=\frac{1+1.5 \phi(1+S(\phi))}{1-\phi(1+S(\phi))},
$$

with $S(\phi)=\phi+\phi^{2}-2.3 \phi^{3}$. This formula was used initially by Ladd to fit his simulation data, obtained for densities up to $\phi=0.45$ using a hydrodynamic force multipoles method [13]. However, it applies to even larger volume fractions since it conforms also with our ASD simulation data, and the ones of Sierou and Brady [29], which have been obtained for densities up to $\phi \approx 0.6$. Note that equation (9) reduces to the exact Einstein limiting law to linear order in $\phi$, however it does not account for the divergence of $\eta_{\infty}$ at random closed packing where $\phi_{r c p} \approx 0.64$.

The short-time viscosity in dilute suspensions of strongly repelling spheres with prevailing two-body HI can be computed from [32]

$$
\frac{\eta_{\infty}}{\eta_{0}}=1+\frac{5}{2} \phi(1+\phi)+\frac{15}{2} \phi^{2} \int_{2}^{\infty} d x x^{2} g(x) J(x)
$$

where the function $J(x)$ accounts for two-body HI. The $(5 / 2) \phi^{2}$ contribution arises from regularizing the integral, which is a summation over induced hydrodynamic force dipoles. The integral involving the radial distribution function, $g(x)$, is at most of order one. In dilute deionized systems, one can approximate the integral using a schematic model for $g(x)$. This leads to the result (see [12] for its derivation)

$$
\frac{\eta_{\infty}}{\eta_{0}} \approx 1+\frac{5}{2} \phi(1+\phi)+7.9 \phi^{3}
$$




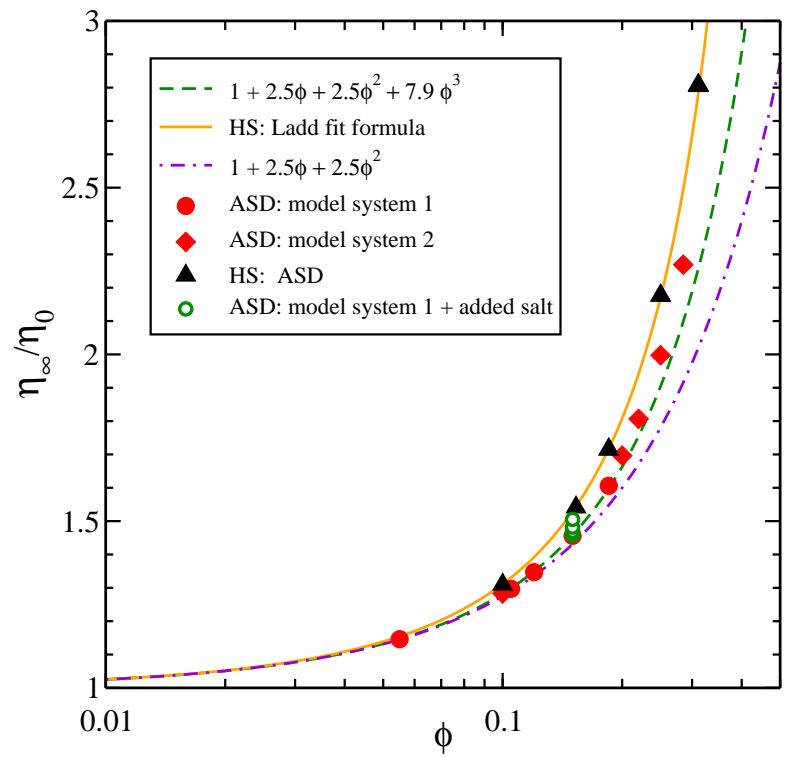

Figure 5. High-frequency limiting viscosity, $\eta_{\infty}$, of two deionized suspensions of charged spheres (circles: $Z=100, a=100 \mathrm{~nm}, L_{B}=5.62 \mathrm{~nm}, n_{s}=0$; diamonds: $\left.Z=70, a=25 \mathrm{~nm}, L_{B}=0.71 \mathrm{~nm}, n_{s}=0\right)$, and of neutral hard spheres, versus $\phi$. Displayed are our ASD simulation data in comparison with the hard-sphere simulation fit formula of Ladd in equation (9), and the expression in equation (11) for deionized suspensions that derives from a schematic model of the pair distribution function.

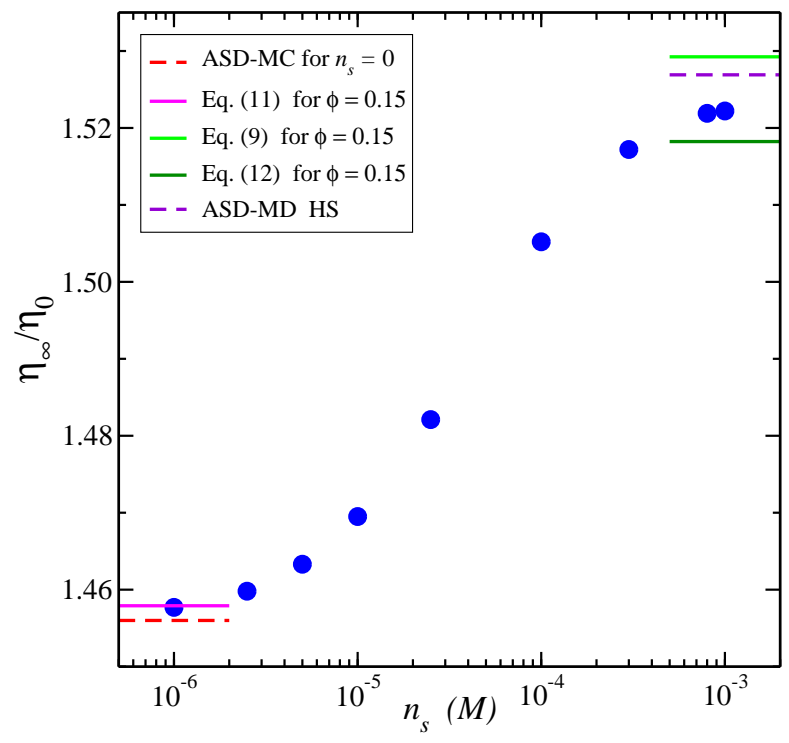

Figure 6. ASD simulation results (circles) on the salt-dependence of the highfrequency limiting viscosity for a charge-stabilized suspension with $\phi=0.15$. 
which includes a correction term $7.9 \phi^{3}$ that describes the viscosity contribution arising from binary particle correlations. It is of cubic order since in deionized systems the peak position of the radial distribution function scales like $\phi^{-1 / 3}[12]$. We can see in figure 5 that this expression conforms rather well with the ASD data on deionized suspensions.

Equation (11) applies to deionized suspensions. Therefore it should be distinguished from the regular virial expansion of $\eta_{\infty}$ existing for colloidal hard spheres. The latter is given by

$$
\eta_{\infty} / \eta_{0}=1+2.5 \phi+5.0023 \phi^{2}+9.09 \phi^{3}+\mathcal{O}\left(\phi^{4}\right)
$$

The third-order virial coefficient, 9.09, which stems from the triplet correlations, has been derived only very recently in [33]. The fourth and higher-order coefficients are not known analytically to date. The third-order virial result for hard spheres is applicable only for $\phi \leq 0.3$. At larger $\phi$, the increase in $\eta_{\infty}$ is underestimated. The hard-sphere virial result in equation (12), and the zero-salt prediction for charged spheres in equation (11), are depicted as horizontal bars for the system in figure 6 .

Even though in the present section we are concerned with short-time properties only, a few remarks are in order here regarding the long-time (static) viscosity $\eta=\eta_{\infty}+\Delta \eta$. For strongly correlated particles, $\eta$ can be substantially larger than its short-time part, since the viscosity contribution $\Delta \eta$ coming from shear-flow-induced, non-instantaneous stress relaxations is dominant $[1,34]$. At equal concentration, the $\Delta \eta$ of charged spheres is larger, since charged spheres resist more strongly a flow-induced distortion of their microstructure. Even though a calculation of long-time properties such as $\eta$ has become feasible for charged spheres using the ASD simulation technique, it will be exceedingly more time-consuming than a short-time calculation. Therefore, we will leave long-time simulations of charge-stabilized spheres to a future study.

\subsection{Generalized Stokes-Einstein relations}

There is considerable interest in identifying generalized Stokes-Einstein relations between diffusion and viscoelastic properties, since these are fundamental to microrheological experiments with their widespread applications to biological systems. Provided a proposed GSE relation is valid, a rheological experiment can be performed more easily, and for smaller samples, using scattering techniques.

Out of various GSE proposals, we will explore the following three short-time relations $[30,34,35,36]$

$$
\begin{aligned}
D_{s}(\phi) & =\frac{k_{B} T}{6 \pi \eta_{\infty}(\phi) a} \\
D_{r}(\phi) & =\frac{k_{B} T}{8 \pi \eta_{\infty}(\phi) a^{3}} \\
D\left(q_{m} ; \phi\right) & =\frac{k_{B} T}{6 \pi \eta_{\infty}(\phi) a} .
\end{aligned}
$$

These expressions relate the high-frequency limiting viscosity to $D_{s}, D_{r}$, and to the short-time cage diffusion function $D\left(q_{m}\right)$, respectively. In the following, we will expose 


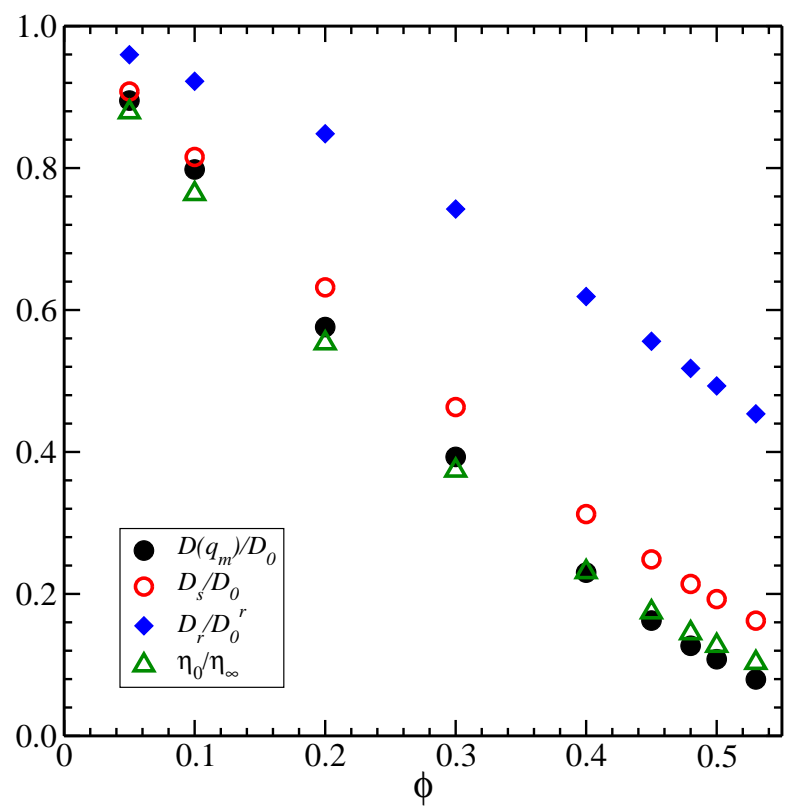

Figure 7. ASD simulation test of the generalized SE relations in equations (13 15) for the hard-sphere case. Symbols: ASD simulation data for $D\left(q_{m}\right) / D_{0}, D_{s} / D_{0}$, $D_{r} / D_{0}^{r}$ and $\eta_{0} / \eta_{\infty}$ as indicated in the legend.

these relations to a stringent test using the ASD simulation technique.

For hard spheres, the $\phi$-dependence of $D_{s} / D_{0}, D_{r} / D_{0}$ and $\eta_{0} / \eta_{\infty}$ is shown in figure 7. It is noted there that the second GSE relation considered here, equivalent to $D_{r} / D_{0}^{r}=\eta_{0} / \eta_{\infty}$, that involves the short-time rotational diffusion coefficient $D_{r}$ with infinite dilution value $D_{0}^{r}=k_{B} T /\left(8 \pi \eta_{0} a^{3}\right)$, is strongly violated for non-zero concentrations. The GSE relation for $D_{s}$, on the other hand, is less strongly violated, but the inequality $D_{s} / D_{0}>\eta_{0} / \eta_{\infty}$ or, likewise, $D_{s}>k_{B} T /\left(6 \pi \eta_{0} a\right)$ is obeyed for all nonzero concentrations. This corresponds to a monotonic and essentially linear increase of $\left(\eta_{\infty} / \eta_{0}\right) \times\left(D_{s} / D_{0}\right)$ in $\phi$, described up to $\phi=0.5$ approximately by the form $1+0.67 \phi$.

Using standard Stokesian Dynamics and ASD simulations, Brady and coworkers have determined the $\eta_{\infty}, D_{s}$ and the static viscosity $\eta$ of hard spheres for densities even above the freezing point, on assuming that the dispersion can be maintained in a noncrystalline, disordered and metastable state up to random close packing. Their data for $D_{r}$ and $\eta_{\infty}$ are not included in figure 7 since, in the liquid phase regime considered here, they fully agree with our simulation results. For values $\phi>0.6$, where random closed packing is approached, Sierou and Brady [29] observe a linear scaling of $D_{s}(\phi)$ as function of the inverse high-frequency viscosity, which gives evidence that $D_{s} \propto 1 / \eta_{\infty}$ in the limit $\phi \rightarrow \phi_{r c p}$. Thus, their simulations suggest that $D_{s}$ vanishes at $\phi_{r c p}$ in a manner inversely proportional to $\eta_{\infty}$. For volume fractions below freezing, however, the physical mechanisms of self-diffusion and viscosity are clearly distinguishable from each other, and the GSE relation for $D_{s}$ is violated to some extent, as noted in figure 7 and quantified by $D_{s}(\phi) / D_{0} \approx(1+0.67 \phi) \times \eta_{0} / \eta_{\infty}(\phi)$.

For hard spheres, the GSE relation involving $D\left(q_{m}\right)$ is satisfied overall to a 


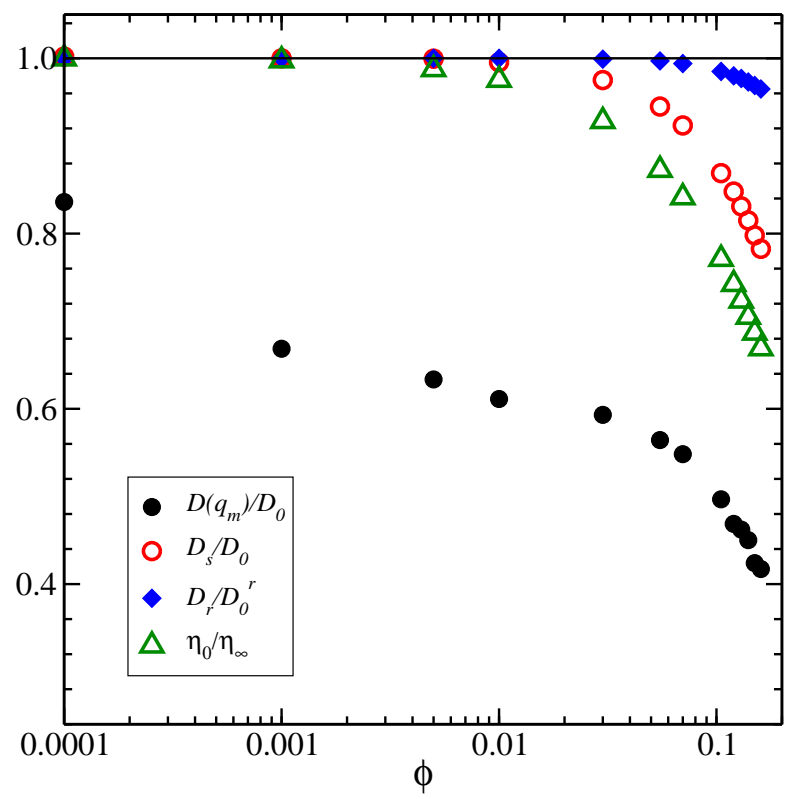

Figure 8. Same as in figure 7, but for a deionized suspension of charged spheres.

remarkably good degree of accuracy. Interestingly enough, and opposite to neutral spheres, the GSE relation for the $D\left(q_{m}\right)$ of charged spheres at zero salinity is the most strongly violated one (see figure 8), whereas the GSE relation for $D_{s}$ still works decently well. This reflects the point raised earlier in our discussion of figure 3 , that different from $\eta_{\infty}$ and $D_{s}, D\left(q_{m}\right)$ is highly sensitive to system parameters such as the particle charge and ionic strength, through its explicit dependence on $S\left(q_{m}\right)$. Note here again the sharp drop in $D\left(q_{m}\right)$ at very small $\phi$ which reflects a correspondingly sharp increase in $S\left(q_{m}\right)$.

As can be noted from figure 8, the relation for $D_{r}$ in charge-stabilized systems is violated to a lesser extent than in the neutral-sphere case. We argue that the GSE relation involving $D_{r}$ is poorer than the one for $D_{s}$, owing to the shorter range of the hydrodynamic mobility tensor associated with rotational self-diffusion. We point out that, according to the ASD data, the ordering relation $D_{s}>k_{B} T /\left(6 \pi \eta_{\infty} a\right)$ is valid both for deionized suspensions and neutral hard spheres, in agreement with experiments of Bergenholtz et al., where $\eta_{\infty}$ and $D_{s}$ have been measured for various ionic strengths and concentrations [30].

For the deionized suspensions in figure 8 one might try to relate the shorttime transport coefficients to an effective volume fraction defined, say, as $\phi_{\text {eff }}=$ $\phi \times(1+1 / \kappa a)^{3}$ corresponding to the effective particle radius $a+\kappa^{-1}$, with $\kappa$ given in equation (2). However, the electric double layers in deionized suspensions are so extended that the so defined $\phi_{\text {eff }}$ can attain unphysically large values due to the nonlinear $\phi$-dependence of $\kappa$ at low salinity. The form of $\phi_{e f f}(\phi)$ is strongly dependent on system parameters, in particular on the residual microionic concentration due to the selfdissociation of solvent (water) molecules. Assuming zero self-dissociation would result 
even in a non-monotonic $\phi$-dependence of $\phi_{\text {eff }}$ where two bare volume fractions are mapped on a single effective one. These considerations illustrate that the concept of an effective volume fraction is not useful in describing the diffusion, and the microstructure (see [11]), of strongly repelling charged particles with thick double layers.

The deviations from the short-time GSE relations can be intuitively rationalized by introducing apparent slip-stick parameters, $\nu$, defined by

$$
\begin{aligned}
D & =\frac{k_{B} T}{6 \pi \eta_{0} a\left(1+\nu_{s, c} \Delta \eta\right)} \\
D_{r} & =\frac{k_{B} T}{8 \pi \eta_{0} a^{3}\left(1+\nu_{r} \Delta \eta\right)},
\end{aligned}
$$

with $\Delta \eta=\eta_{\infty} / \eta_{0}-1$ and $D$ used as an abbreviation for $D_{s}$ and $D\left(q_{m}\right)$. Here, $\nu_{s}, \nu_{r}$ and $\nu_{c}$ are the slip-stick parameters for translational and rotational self-diffusion, and for cage diffusion, respectively. The parameters are determined from

$$
\nu_{s, c, r}(\phi)=\frac{D(\phi=0) / D(\phi)-1}{\eta_{\infty}(\phi) / \eta_{0}-1},
$$

with $D=D_{s}, D\left(q_{m}\right)$ and $D_{r}$, respectively. The friction term in the denominator of equation (16) has been split into a part due to the solvent alone, which sticks to the surface of the large colloidal spheres, and an additional frictional part originating from the direct and hydrodynamic interactions between the spheres. Since the spheres are all of equal size, there is no reason to expect a stick boundary condition to be valid for the interaction frictional part, which would correspond to $\nu=1$. The description of the host suspension surrounding a colloidal probe sphere as a continuous medium without local inhomogeneities, characterized by $\eta_{\infty}$, is strictly valid only when the probe sphere is much larger than the host spheres. In fact, for a colloidal tracer sphere of radius $a$ immersed in a host suspension of neutral spheres of radius $a_{H}$, the slip-stick parameters of the tracer increase from 0 for $a / a_{H} \rightarrow 0$ (i.e., zero excess friction) up to the value 1 for $a / a_{H} \rightarrow \infty$, in accord with the expectation that the GSE relations become exactly valid only in the continuum limit of the host dispersion (c.f., $[35,36]$ ).

In figures $9 \mathrm{a}$ and $9 \mathrm{~b}$, we show the slip-stick parameters for hard spheres and deionized charged spheres, respectively, as obtained from the ASD data in figures 7 and 8 using equation (17). The average slip-stick parameter $\nu_{s}$ for hard spheres is close to $2 / 3$ (dashed line). The excess frictional part due to interactions is thus well described for hard spheres by the perfect slip form $4 \pi\left(\eta_{\infty}-\eta_{0}\right) a$. For rotational self-diffusion, $\nu_{r} \approx 0.20$ (dashed line), i.e., the interaction friction is less pronounced in the case of rotational self-diffusion so that $D_{r} / D_{0}^{r}>D_{s} / D_{0}$. The parameter $\nu_{c}$ characterizing cage diffusion is close to 1 in the case of hard spheres for all values of $\phi$. For deionized suspensions of charged spheres, however, super-stick values of $\nu_{c}$ are observed throughout that are substantially larger than 1 for all concentrations considered. This is reflected in $D\left(q_{m}\right) / D_{0}<\eta_{0} / \eta_{\infty}$ (see figure 8) and indicates, for the cage diffusion of charged spheres at low salinity, the failure of the continuum picture for the equally-sized host suspension. According to equation (17) the parameters $\nu_{r, s}$ for charged particles at very small $\phi$, where $D_{s} \approx D_{0}, D_{r} \approx D_{0}^{r}$ and $\eta_{\infty} \approx \eta_{0}$, are very sensitive to statistical errors 

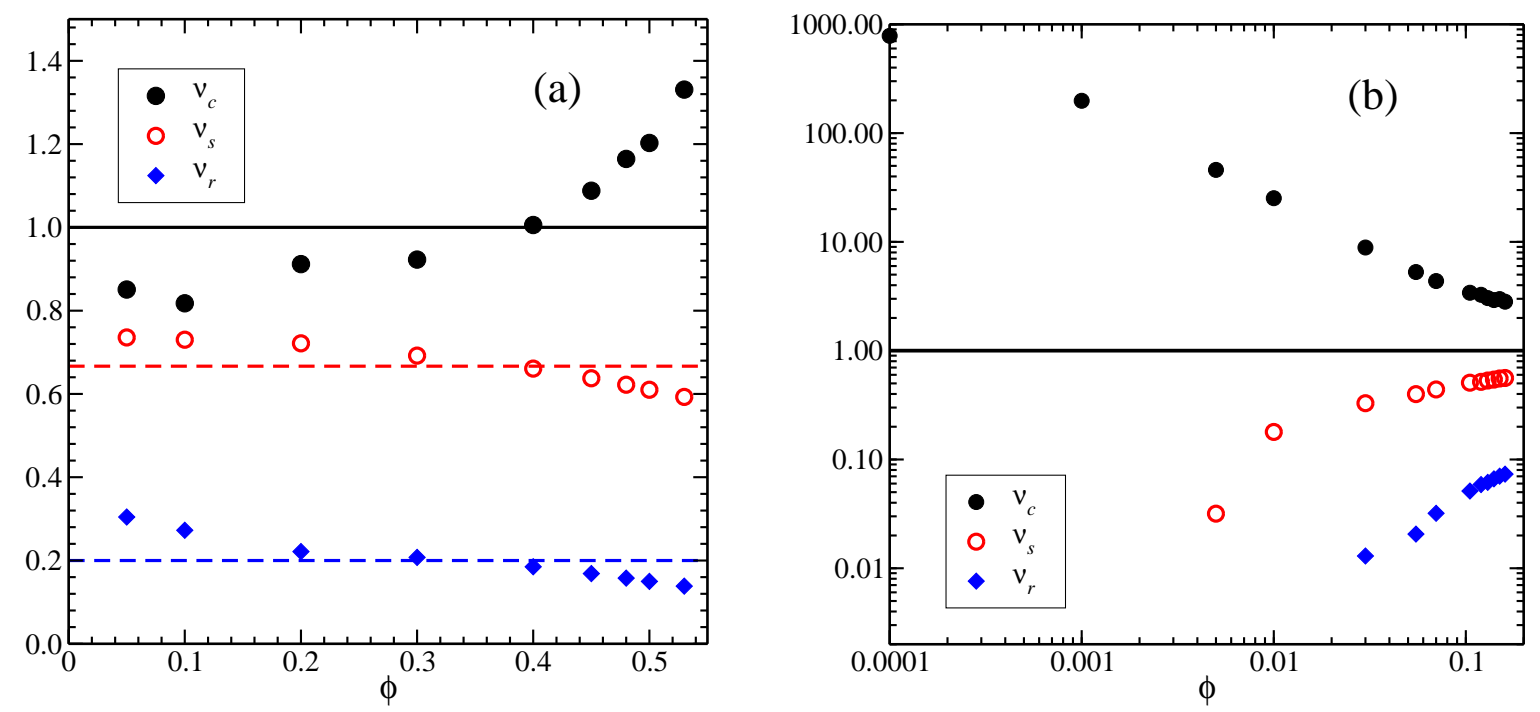

Figure 9. Apparent slip-stick coefficients $\nu_{s}, \nu_{r}$ and $\nu_{c}$ characterizing the interaction friction contribution to the translational and rotational self-diffusion, and the cage diffusion coefficient, respectively. Shown are ASD results for (a): hard spheres, and (b): a deionized suspension of charged spheres. The dashed lines in (a) mark the volume fraction averaged values $\left\langle\nu_{s}\right\rangle \approx 2 / 3$ and $\left\langle\nu_{r}\right\rangle=0.20$, respectively.

in the ASD data. Therefore, in figure 9b these values have not been included. Note further that the strong deviations of the slip-stick parameters of charged spheres from 1 become smaller for increasing values of $\phi$.

In summary, a major conclusion of our simulation study is that the approximate validity of (short-time) GSE relations depends in general strongly on the range of the pair potential. Finally, we mention that a test of corresponding long-time GSE relations has been made in [34], on the basis of an idealized mode-coupling approximation, with HI accounted for in an ad hoc fashion using a short-time hydrodynamic rescaling prescription, applicable unfortunately to neutral hard spheres only. A long-time simulation analysis of the dynamics of charge-stabilized colloidal spheres with a full account of the HI remains as a major challenge to a future study. 


\section{Electrokinetic Effects on Colloidal Long-time Diffusion}

The electrolyte friction effect is an additional friction experienced by diffusing charged colloidal particles. This friction results from the relaxation of the electric double layer around a diffusing colloidal particle, as a non-instantaneous response to any disturbance in the spherical symmetry of the double layer. It causes a reduction in the long-time self-diffusion coefficient, $D^{L}$, of a charged colloidal sphere. This coefficient quantifies the long-time slope of the colloidal mean-squared displacement. To correctly describe this dynamic friction effect, one must take account of the colloidal Brownian motion, the presence of further colloidal spheres, the dynamics of the various species of electrolyte ions (microions) and the hydrodynamic interactions between all aforementioned particles. A versatile method for calculating the long-time selfdiffusion coefficient of repulsive charged colloidal spheres, based on the Primitive Model where all ionic species are treated equally and individually as uniformly charged hard spheres immersed in a structureless solvent, and the many-body Smoluchowski diffusion equation, has been developed in a couple of papers by the present authors [2, 37]. This method invokes a simplified mode-coupling scheme for mixtures of Brownian spheres [38, 39, 40, 41], and it accounts for the long-distance part of the HI between all ionic species in the form of the so-called Rotne-Prager approximation [42]. The detailed derivation of this method, and a thorough discussion of the approximations involved in its derivation, has been given elsewhere $[2,37]$ and will not be repeated here. To our knowledge, this is the first statistical mechanical scheme describing the dynamic electrolyte influence on the long-time self-diffusion of charged colloids in non-dilute suspensions with an account of the inter-ionic HI.

Different from the solvent, which is described as a structureless continuum, the hydrated microions are treated here as separate bodies since the hydrated ions are somewhat larger and heavier than the water molecules giving rise to a larger relaxation time of the ionic momenta [43]. In addition, the microions are coupled through their electric charge to the much slower motion of the colloidal macroions. Using the Smoluchowski equation describing overdamped Brownian motion also for the microions is an approximation, in particular when considering very short times in the subpicosecond range where the inertia of the microions matters. In this ultra-short time range, a generalized Fokker-Planck description accounting for the ion momenta would be more appropriate. However, we are interested here in the (long-time) dynamics of colloidal macroions which takes place on time scales that are many orders of magnitude larger, and which is influenced by the microions through their cooperative motion only. Treating the electrolyte ions as individual particles allows to account for microionic correlation and finite size effects on the colloid dynamics, which can be of importance for strongly charged and very small colloids.

According to our scheme, the long-time self-diffusion coefficient of charge-stabilized colloidal spheres at non-zero colloid concentration $n$ is given by the Stokes-Einstein-like 
relation [2],

$$
\frac{D^{L}}{D^{0}}=\left[1+\frac{\Delta \zeta^{E F}}{\zeta^{0}}+\frac{\Delta \zeta^{C F}}{\zeta^{0}}\right]^{-1}
$$

that includes, in addition to the single-sphere solvent friction part, $\zeta^{0}=k_{B} T / D^{0}$, an electrolyte friction (EF) and a colloid friction $(\mathrm{CF})$ contribution. The colloid friction contribution arises from the microion-averaged electrosteric and hydrodynamic interactions between the colloidal particles. Different from section 2, we use here superscripts to label the single-sphere diffusion coefficient, $D^{0}$, of a single colloidal sphere and the long-time self-diffusion coefficient, $D^{L}$, of interacting spheres. The subscript $i$ is used in the following to label the various microionic species.

\subsection{Electrolyte and colloid friction}

In [2], an expression has been derived for the electrolyte friction contribution, in which the ionic species have equal mobilities. In the present work, our scheme for calculating $D^{L}$ is extended to electrolyte ion species of unequal mobilities and valencies. If the microionic species have unequal free mobilities which are, however, much larger that the free mobility of the colloidal spheres, the expression for the electrolyte friction contribution derived in [2] is generalized to

$$
\frac{\Delta \zeta^{E F}}{\zeta^{0}}=\frac{2}{3 \pi^{2}} \sum_{i=1}^{m} n_{i} \frac{D^{0}}{D^{0}+D_{i}^{0}} \int_{0}^{\infty} d k k^{2}\left\{\frac{S(k) h_{c i}^{d}(k)-h_{c i}(k) H(k)}{S(k)+H(k)}\right\}^{2},
$$

where the $D_{i}^{0}$ are the free diffusion coefficients of the electrolyte ion species $i \in\{1, \ldots, m\}$ of number density $n_{i}, S(k)$ and $H(k)$ are the static structure factor and hydrodynamic function of the colloid species, and $h_{c i}(k)$ and $h_{c i}^{d}(k)$ are the partial total correlation and partial distinct hydrodynamic functions between the colloidal spheres and the electrolyte ion of species $i$, respectively. It is interesting to note in this expression that the electrolyte friction contribution from each of the $m$ microionic species are decoupled, and that the hydrodynamic interactions between the microionic species does not appear. In fact, this expression is an approximation applicable to the case when $\delta_{i}=D^{0} /\left(D^{0}+D_{i}^{0}\right)$ is small for all $i$. The higher order terms in the expansion in the $\delta_{i}$ do include the microion-microion hydrodynamic interactions, but since these prefactors are small in colloidal systems, they are ignorable.

In the limiting case that the colloidal species is infinitely dilute, $S(k)$ and $H(k)$ become both equal to 1 , so that equation (19) reduces to

$$
\frac{\Delta \zeta^{E F}}{\zeta^{0}}=\frac{1}{6 \pi^{2}} \sum_{i=1}^{m} n_{i} \frac{D^{0}}{D^{0}+D_{i}^{0}} \int_{0}^{\infty} d k k^{2}\left\{h_{c i}(k)-h_{c i}^{d}(k)\right\}^{2} .
$$

Note here that the Rotne-Prager form of the colloid-microion HI is exact in the case of a single forced colloidal sphere in a suspension of pointlike microions. For zero colloid concentration, $\Delta \zeta^{C F}=0$, and the electrolyte friction is the only additional non-solvent source of friction on the colloids in the long time regime. Using the mean spherical approximation (MSA) for the colloid-microion correlation functions, so that 
$h_{c i}=h_{c}^{H S}+z_{i} h_{c}^{E L}$ and $h_{c i}^{d}=h_{c}^{d H S}+z_{i} h_{c}^{d E L}$, the electrolyte friction expression further simplifies to

$$
\frac{\Delta \zeta^{E F}}{\zeta^{0}}=\frac{\kappa^{2}-\kappa_{m}^{2}}{24 \pi^{3} L_{B}} \int_{0}^{\infty} d k k^{2}\left\{h_{c}^{E L}(k)-h_{c}^{d E L}(k)\right\}^{2}
$$

where $\kappa_{m}^{2}=4 \pi L_{B} \sum_{i=1}^{m} n_{i} \frac{D_{i}^{0}}{D^{0}+D_{i}^{0}} z_{i}^{2}$ and $\kappa^{2}=4 \pi L_{B}\left(\sum_{i=1}^{m} n_{i} z_{i}^{2}+n|Z|\right)$, with $Z$ denoting the valency of a colloidal sphere and $n$ the colloid concentration $(n=0$ in equations (20) and (21)).

There is no contribution to the electrolyte friction from excluded volume interactions between the colloidal sphere and the electrolyte ions because the flow field created by a single colloidal sphere advects point particles along the streamlines of the colloid [2]. Equation (21) is reminiscent of the Onsager limiting law for dilute electrolytes (without colloids) which states that the additional friction on an electrolyte ion of charge number $z$ due to the other ones is [44]

$$
\frac{\Delta \zeta^{E F}}{\zeta^{0}}=\frac{L_{B} z^{2}}{3}\left[\kappa-\kappa_{m}\right] .
$$

In the case that the colloidal sphere is uncharged, there is no additional friction from the electrolyte ions, since the friction in equation (21) is proportional to the square of the colloid charge. There is a residual friction, however, when considering a dense colloidal suspension since then the flow field around a neutral colloidal spheres is affected by the presence of the other colloids, and the flow does not simply advect the small pointlike ions any more. However, this residual electrolyte friction is very small and results only from excluded volume interactions, so that it would be present even when both the colloids and the electrolyte particles were uncharged.

We note here that the colloid friction contribution, which arises from the static and hydrodynamic interactions among the colloids themselves, is given in our scheme by [2]

$$
\frac{\Delta \zeta^{C F}}{\zeta^{0}}=\frac{1}{6 \pi^{2} n} \int_{0}^{\infty} d k k^{2} \frac{[S(k)-H(k)]^{2}}{[S(k)+H(k)]} .
$$

Although there is no dynamic influence of the electrolyte ions on the colloid friction, this friction contribution is indirectly influenced through static colloid-microion and microion-microion correlations that describe the static screening of the bare Coulomb interactions between the colloids.

\subsection{Charge- and mobility asymmetric electrolytes}

In the following, we quantify the effect on the electrolyte friction originating from an asymmetry between the electrolyte ions. This asymmetry can be either in the form of a charge or mobility asymmetry. The example system discussed here is a simplifying colloid model of short 20 base-pair-long DNA oligomers in aqueous dispersion [45]. We treat these biological particles as spheres of radius $a=3.4 \mathrm{~nm}$, and charge number $Z=-42$. Due to the small size of the biological particles, the electrolyte friction will make a rather large contribution. The electrolyte ions and surface-released counterions 


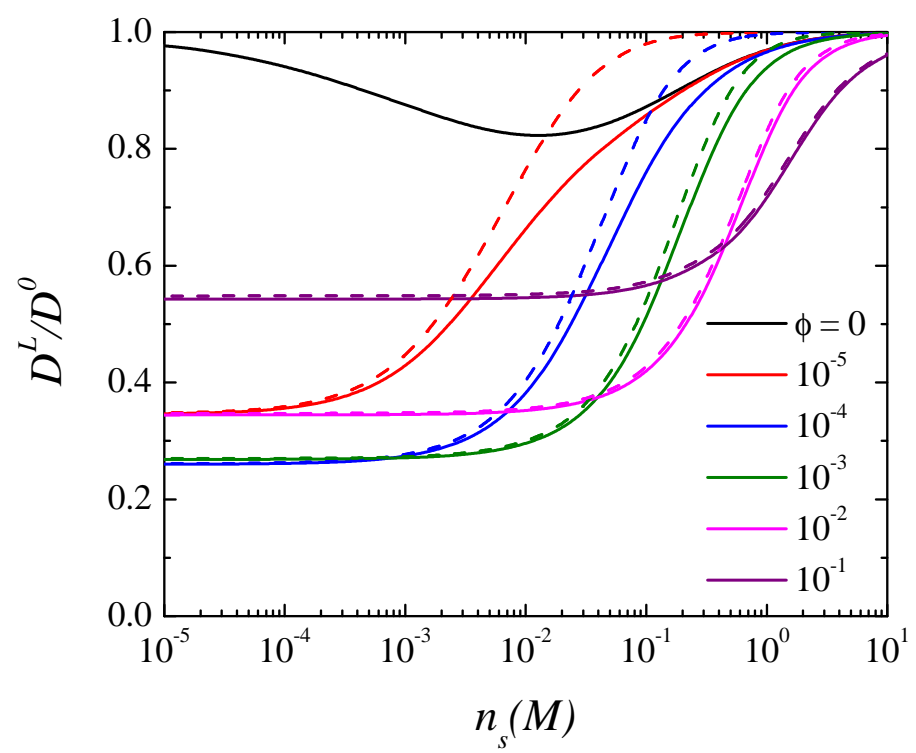

Figure 10. Long-time colloidal self-diffusion coefficient, $D^{L}$, of the simplifying spherical DNA fragment model, as a function of added monovalent salt concentration. The solid lines include the electrolyte friction contribution, the dashed lines contain only the colloid friction contribution.

are treated as pointlike, and the static inter-ionic correlation functions required as input to our scheme, are calculated using the analytic MSA solution for the Primitive Model. The MSA accounts for the excluded volume of the colloids and preserves the exact zeroth and second order moments conditions of local charge neutrality and charge oscillations valid in equilibrium.

Taking the solvent to be water at $20^{\circ} \mathrm{C}$ corresponding to a Bjerrum length $L_{B}=0.71 \mathrm{~nm}$, and the average radius of monovalent microions to be approximately $2.5 \AA$, we have that the free diffusion coefficients of the microions and the colloids are $D_{ \pm}^{0}=1.0 \times 10^{-9} \mathrm{~m}^{2} / \mathrm{s}$ and $D^{0}=0.6 \times 10^{-10} \mathrm{~m}^{2} / \mathrm{s}$ respectively. Moreover, we assume for simplicity that the counterions dissociated from the DNA surfaces (surface-released counterions) are of the same species as the counterions of the dissociated added salt.

In figure 10, we show the calculated long-time diffusion coefficient, $D^{L}$, of our DNA fragment model as a function of added salt concentration, $n_{s}$, for a series of DNA volume fractions as indicated. The solid lines arise from including both the colloid and electrolyte friction contributions, and the dashed lines from including only the colloid friction. The effect of electrolyte friction is seen to be most important at low volume fractions. It is essentially non-visible at higher volume fractions.

The minimum in the diffusion constant for $\phi=0$ is due to electrolyte friction alone and occurs at intermediate salt concentrations where $\kappa^{-1}$ is of the order of the sphere radius. It is due basically to the colloid-microion HI. As shown in [2] for macroions with fixed surface electric potential, ignoring this dynamic interaction in model calculations 


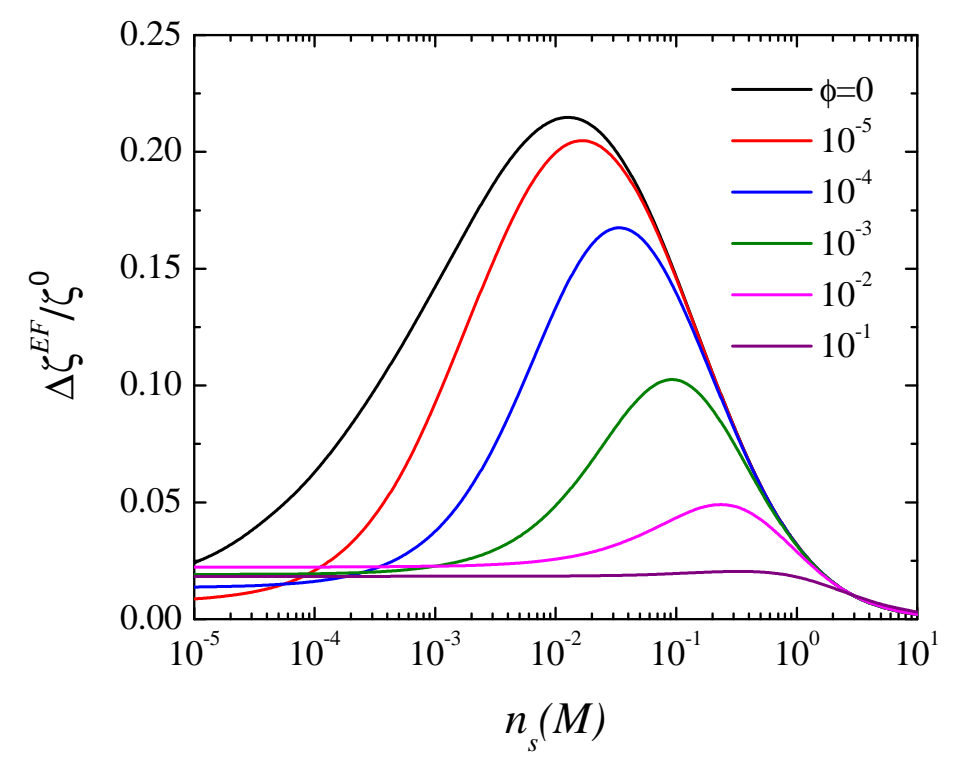

Figure 11. Electrolyte friction contribution for the spherical DNA fragment model, with $a=3.4 \mathrm{~nm}$ and $Z=-42$, as a function of added monovalent salt concentration, with all microions of equal mobility.

may result in unphysical predictions. A similar minimum in the salt-dependence of the translational $D^{L}$ is found both experimentally and theoretically also for rodlike charged particles [46, 47], and charged semi-flexible polymers such as double-stranded DNA fragments [48].

Figure 11 displays explicitly the colloidal electrolyte friction contribution due to added monovalent salt ions $(1: 1$ electrolyte) of equal mobilities. The electrolyte friction has a maximum at intermediate salt concentrations due to the interplay between the relaxation of the microions in the electric double layer and the extent of the double layer as quantified by the screening length $\kappa^{-1}$. The electrolyte friction reduces with added colloid concentration due to the increase in the electrolyte background concentration, which lowers the distortion of the electric double layers in relation to the background electrolyte concentration. It is because of this that the long-time self-diffusion in dense systems can be accounted for almost entirely through the colloidal friction contribution $\Delta \zeta^{C F}$.

In figure 12, the electrolyte friction contribution is shown for the two cases when either the counter- or co-ions of a $1: 1$ electrolyte have twice the mobility of the opposing microions. Again the same overall trends in the electrolyte friction can be seen in these results, i.e., that the electrolyte friction has a peak at intermediate added salt concentrations and that it reduces significantly with increased colloid concentration. However, the effect of increasing the mobility of one of the electrolyte ion species is to decrease the electrolyte friction overall due to the decrease in the relaxation time of the electric double layer. Furthermore, at zero colloid concentration, there is no 


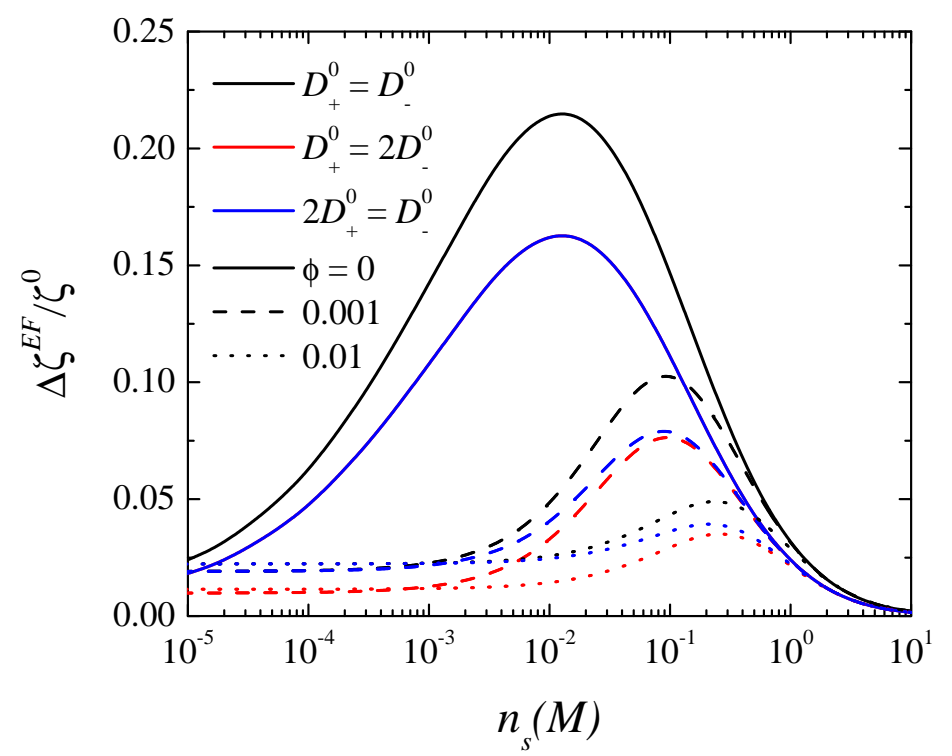

Figure 12. Reduced electrolyte friction contribution for the spherical DNA fragment model, with $a=3.4 \mathrm{~nm}$ and $Z=-42$, as a function of added monovalent salt concentration, with differing microionic free mobilities as indicated by the differing colors. Three sets of data are considered for $\phi=0$ (solid lines), $10^{-3}$ (dashed lines) and $10^{-2}$ (dotted lines), in order from largest to lowest.

difference in whether the counter- or co-ions have the larger mobility since the surfacereleased (positive valued) counterions are also at zero concentration and make therefore no contribution to the friction. Indeed, in the two cases considered, that is counteror co-ions with doubled mobility, there is no difference in the electrolyte friction even for non-zero colloid concentration when the added salt ions dominate over the surfacereleased counterions. When the surface-released counterions dominate (low-salt regime) then there is a difference in the electrolyte friction. This is due to the fact that in this case there are very few coions as compared to the counterions, so that it is unimportant what mobility they have.

It is important to note that when the mobility of microions is increased, only the electrolyte friction is affected due to the decrease in the microionic relaxation time. The colloid friction described by equation (23), however, is unaffected by the mobilities of the electrolyte ions.

In figure 13, the electrolyte friction contribution is shown for the two cases when the counter- or co-ions are divalent, i.e., we consider here the electrolyte friction of DNA 'spheres' in a $z_{+}: z_{-}=2: 1$ and $1: 2$ electrolyte solution in comparison to a 1 : 1 electrolyte system, in dependence on the number concentration, $n_{s}$, of salt ion pairs. Interestingly enough, the magnitude of the electrolyte friction peak is unaffected by the valency change in the electrolyte ions, however its location is shifted to lower added salt concentration. This behavior can be attributed to the fact that the extension of the electric double layer around a DNA 'sphere' is reduced when the charge of the electrolyte ions is increased for a fixed $n_{s}$. This is more easily seen in figure 14 where the 


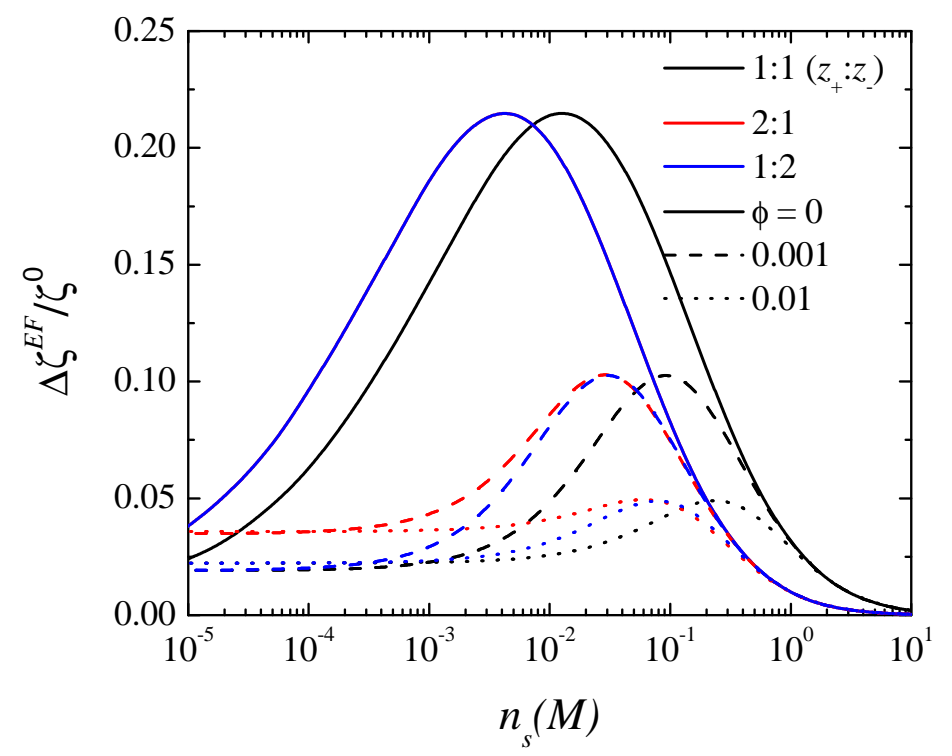

Figure 13. Electrolyte friction contribution for the spherical DNA fragment model, with $a=3.4 \mathrm{~nm}$ and $Z=-42$, as a function of added salt concentration, with differently charged electrolyte ions as indicated by the differing colors. There are three sets of data for $\phi=0,10^{-3}$ and $10^{-2}$, in order from largest to lowest.

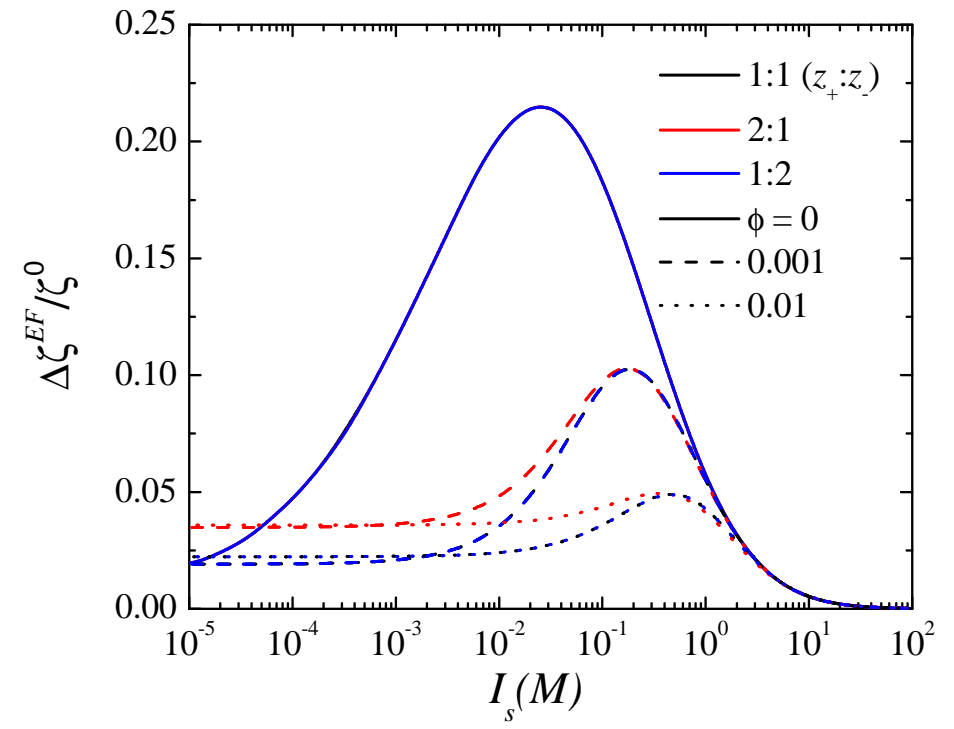

Figure 14. Same as in figure 13, but plotted versus ionic strength $I_{s}$.

electrolyte friction contribution is plotted against the ionic strength of the added salt, $I_{s}=\sum_{i=1}^{2} n_{i} z_{i}^{2}$ with $n_{1}=n_{s} z_{+}$and $n_{2}=n_{s}\left|z_{-}\right|$, measured in mol/l. In this plot, the peaks in the electrolyte friction coincide for all three electrolyte solutions considered. 
At zero DNA concentration $(\phi=0)$, it does not matter which microion species is of higher charge. At very low salt concentration and non-zero $\phi$, however, when the surface-released counterions dominate, an enlarged valency of the counterions does cause a higher electrolyte friction. This can be ascribed to the fact that in this case the electric double layer has twice the extension of a corresponding system with monovalent counterions.

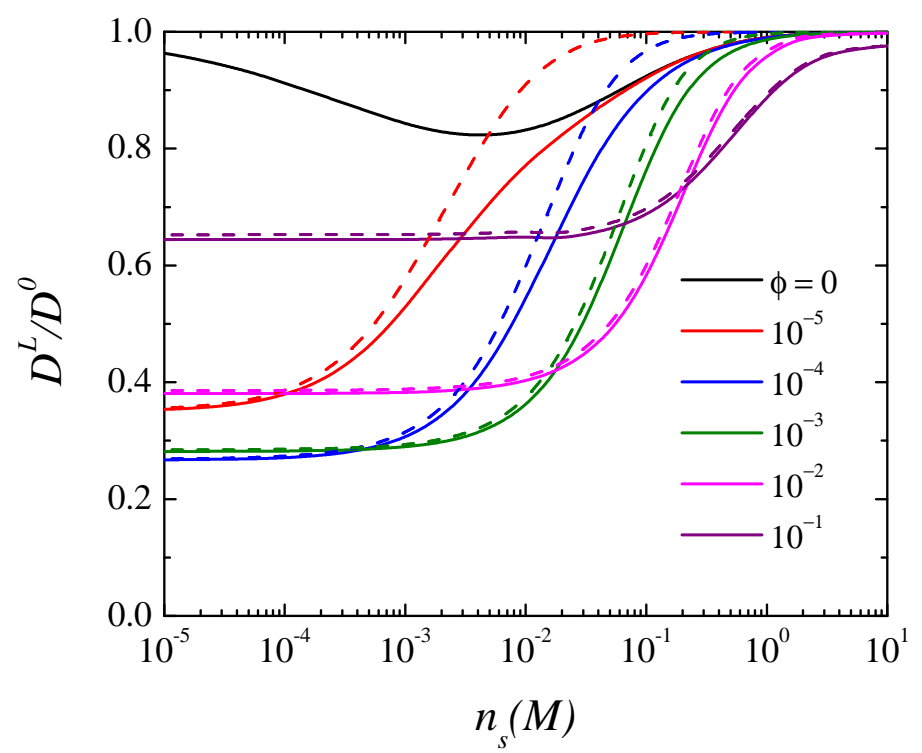

Figure 15. Long-time self-diffusion coefficient of the spherical DNA fragment model as a function of added 2:1 salt concentration with divalent counterions. The solid lines include the electrolyte friction contribution, dashed lines contain only the colloid friction contribution.

Finally, in figure 15, we show the long-time self-diffusion coefficient of DNA fragments in a $2: 1$ electrolyte solution. This should be compared to figure 10 showing the $D^{L}$ for a $1: 1$ electrolyte. There is overall only a small difference in the $D^{L}$ of the two cases owing to the dominance of the colloid friction contribution. However, the increase in $D^{L}$, i.e., the decrease in the total friction, that occurs with increasing salt concentration, appears at lower salt concentrations for a $2: 1$ electrolyte solution due to increased screening caused by the divalent electrolyte ions. 


\section{Summary}

In the first part of this paper, we have presented numerous simulation results for short-time dynamic properties of charge-stabilized suspensions including selfdiffusion coefficients, hydrodynamic function and high-frequency-limiting viscosity. The calculations have been made using an extension of the ASD simulation method to the OMF model of charged colloidal spheres.

The range of validity and the accuracy of various short-time GSE relations have been examined. The assessment of the quality of these relations is a necessary prerequisite for the experimentalist interested in their use for data interpretation, for instance in the context of microrheological experiments. Our simulation results have shown that rotational self-diffusion and, to a lesser extent, translational self-diffusion is faster than predicted by the corresponding GSE relations. We have found that the amount by which a GSE relation is violated depends sensitively on the range of the electrostatic repulsion. A case in point is the GSE relation for the cage diffusion coefficient which applies reasonably well to neutral hard spheres, but is strongly violated in the case of deionized suspensions. The general trends in the concentration and salt dependence of $\eta_{\infty}$ (and $D_{s}$ ), predicted by our ASD simulations, are in agreement with the experimental findings on charge-stabilized dispersions.

The ASD simulation study of isobestic points and, more specifically, the calculation of diffusion properties at wave numbers where $S(q)=1$, has allowed us to quantify the experimental error made in determining $D_{s}$ through dynamic light scattering measurements at such points. In our simulations, the error is less than ten percent. This finding is of relevance to scattering experiments on numerous colloidal systems where the large- $q$ regime is not accessible experimentally, and where partial index matching techniques can not be applied.

In the second part of the paper, the multi-component Primitive Model model has been used to explore the dynamic influence of salt ions on the colloidal long-time selfdiffusion in non-dilute suspensions. For this purpose, we have developed a simplified mode-coupling scheme that accounts for the far-field part of the HI between all ionic species, and treats the colloid and electrolyte friction contributions to $D^{L}$ on equal footing. A very convenient, but approximate feature of the mode-coupling scheme is that dynamic transport properties are expressed in terms of static pair correlation functions for which a variety of well-documented solution schemes exist. Microionic correlation effects like quasi-condensation of counterions and overcharging enter into the dynamics through these static correlation functions.

In the present calculations, the microions have been considered as pointlike in comparison to the size of the colloidal particles, but they are characterized by finite values of the free diffusion constants. In an earlier study on the electrolyte friction effect on a single colloidal sphere [37], we have found that the finite size of the microions is insignificant for colloid-microion size ratios typically larger than 5 , and we expect that this holds true also in non-dilute suspensions provided the concentration of salt ions is 
not large and the colloids are not very strongly charged.

We have shown that the electrolyte friction contribution to $D^{L}$ becomes insignificant when the mobility difference between the colloidal spheres and the microions is large, and when the concentration in the suspension increases. The second finding is attributed to the enhanced overall homogenization of the electrolyte background with increasing $\phi$. We have extended our mode-coupling scheme to electrolyte ion species of differing mobilities and valencies. This extension has been used to study the effects of doubling or halving the mobility or charge of one of the two microionic species. It has been shown that these asymmetries can influence the electrolyte friction significantly, and that their effect changes in going from the counterion to the salt-dominated regime.

Finally, we note that substantial progress has been made very recently in developing computer simulation and numerical schemes that allow to calculate the electrokinetic transport properties of charge-stabilized colloids at non-zero concentrations with an account of many-body hydrodynamic effects. In the so-called fluid particle dynamics (FPD) method of Tanaka and Araki [49, 50], and in the related smoothed profile method (SPM) of Kim, Nakayama and Yamamoto [51, 52], the solvent and the microions are treated on a coarse-grained level as continuous fields, akin to standard electrokinetic theory. Only the colloids are treated explicitly as particles. The solid colloidal macroions are modeled in the FPD method as undeformable fluid particles of viscosity much higher than the viscosity of the surrounding solvent. In contrast, in the SPM method the sharp boundary between colloids and fluid is replaced by a diffuse interface of finite thickness. In both methods, the particles act on the solvent through continuous body forces in the Navier-Stokes equation rather than through moving boundary conditions. This simplification results in numerically efficient solution schemes which are formulated in simple Cartesian coordinates. Therefore both methods are suitable to simulate dense colloid dispersions. The SPM method has the advantage that larger time increments can be selected since the particles are treated as solids rather than high-viscous fluid droplets [52]. Since the microions in both methods are described in a mean-field way by a continuous charge distribution, local charge ordering effects beyond the PoissonBoltzmann (PB) level are not considered.

Lobaskin, Dünweg et al. [53, 54] have developed a hybrid simulation method where the microions are considered explicitly. To approximate the fluid stick boundary conditions, a tethered network of small particles is wrapped around the surface of a spherical colloidal macroion (raspberry-like structure). The small particles on the colloid surface and the spherical microions are dissipatively coupled through a friction term to a lattice Boltzmann background describing the hydrodynamics of a structureless solvent. The colloid particle and the finite-sized microions are propagated using a Molecular Dynamics algorithm with Langevin thermal noise. This simulation method goes beyond the more mesoscopic FDP and SPM schemes in that it accounts for the discrete nature of the microions, including thus correlations beyond the PB level. This is achieved for the price of a larger numerical effort. The simulation method is restricted to a limited range of macroion-counterion size ratios. Due to the more costly numerics, only a single 
macroion with its cloud of microions in a box with periodic boundary conditions has been considered so far, with finite colloid concentration effects accounted for simply by adjusting the box to colloid size ratio to the given volume fraction.

To date the numerical methods described above have been applied mainly to colloidal electrophoresis. In principle, they can be used also to describe the electrolyte friction effect. In future studies it will be interesting to explore how these methods compare with each other, and with the semi-analytical mode coupling scheme discussed in this paper.

\section{Acknowledgments}

This work was funded by the Deutsche Forschungsgemeinschaft (SFB-TR6, project section B2). A.J.B. also acknowledges financial support from CONICET, FONCYT (PICT 2005-33691), and SECYT-UNC. We gratefully acknowledge discussions with J.K.G. Dhont, J. Gapinski, Ch. Gögelein, and A. Patkowski.

\section{References}

[1] A J Banchio and J F Brady 2003 J. Chem. Phys. 11810323

[2] M G McPhie and G Nägele 2007 J. Chem. Phys. 127034906.

[3] E J W Verwey and J T G Overbeek 1948 Theory of the Stability of Lyophobic Colloids (Amsterdam: Elsevier)

[4] A R Denton 2000 Phys. Rev. E 623855

[5] E Trizac, L Bocquet, M Aubouy and H H von Grünberg 2003 Langmuir 194027

[6] W L Hsin, T Y Wang, Y J Sheng and H K Tsao 2004 J. Chem. Phys. 1215494

[7] E Castaneda-Priego, L F Rojas-Ochoa, V Lobaskin and J C Mixteco-Sanchez 2006 Phys. Rev. E 74051408

[8] S Pianegonda, E Trizac and Y Levin 2007 J. Chem. Phys. 126014702

[9] M G McPhie and G Nägele 2008 submitted

[10] P N Pusey in Liquids, Freezing and the Glass Transition, edited by J-P Hansen, D Levesque and J Zinn-Justin 1991 (Amsterdam: Elsevier) 763-942

[11] G Nägele 1996 Phys. Rep. 272215

[12] A J Banchio and G Nägele 2008 J. Chem. Phys. 128104903

[13] A J C Ladd 1990 J. Chem. Phys. 933484

[14] A J C Ladd, H Gang, J X Zhu and D A Weitz 1995 Phys. Rev. E 526550

[15] P N Segrè, O P Behrend and P N Pusey 1995 Phys. Rev. E 525070

[16] C W J Beenakker and P Mazur 1984 Physica A 126340

[17] C W J Beenakker 1984 Physica A 12848

[18] U Genz and R Klein 1991 Physica A 17126

[19] G Nägele, B Steininger, U Genz and R Klein 1994 Physica Scripta T 55119

[20] B Noetinger 1989 Physica A 1571139

[21] E Overbeck, C Sinn and M Watzlawek 1999 Phys. Rev. E 601936

[22] A J Banchio, J Gapinski, A Patkowski, W Häussler, A Fluerasu, S Saccana, P Holmqvist, G Meier, M P Lettinga and G Nägele 2006 Phys. Rev. Lett. 96138303

[23] J Gapinski, A Patkowski, A J Banchio, P Holmqvist, G Meier, M P Lettinga and G Nägele 2007 J. Chem. Phys. 126104905

[24] D M E Thies-Weesie, A P Philipse, G Nägele, B Steininger and R Klein 1995 J. Colloid Interface Sci. 17643 
[25] D O Riese, G H Wegdam, W L Vos, R Sprik, D Fenistein, J H H Bongaerts and G Grübel 2000 Phys. Rev. Lett. 855460

[26] T Autenrieth, A Robert, J Wagner and G Grübel 2007 J. Appl. Cryst. 40 s250

[27] P N Pusey 1978 J. Phys. A 11119

[28] R A Lionberger and W B Russel $1994 J$. Rheology 381885

[29] A Sierou and J F Brady 2001 J. Fluid Mech. 448115

[30] J Bergenholtz, F M Horn, W Richtering, N Willenbacher and N J Wagner, 1998 Phys. Rev. E 58 R4088

[31] F M Horn, W Richtering, J Bergenholtz, N Willenbacher and N J Wagner 2000 J. Colloid Interface Sci. 225166

[32] W B Russel 1978 J. Fluid Mech. 85209

[33] B Cichocki, M L Ekiel-Jezewska and E Wajnryb 2003 J. Chem. Phys. 119606

[34] A J Banchio, G Nägele and J Bergenholtz 1999 J. Chem. Phys. 1118721

[35] G H Koenderink, H Zhang, D G A L Aarts, M P Lettinga, A P Philipse and G Nägele 2002 Faraday Discuss. 123335

[36] G Nägele 2003 J. Phys.: Condens. Matter 15 S407

[37] M G McPhie and G Nägele 2004 J. Phys.: Condens. Matter 16 S4021.

[38] G Nägele and J Bergenholtz 1998 J. Chem. Phys. 1089893

[39] G Nägele and J K G Dhont 1998 J. Chem. Phys. 1089566

[40] G Nägele, J Bergenholtz and J K G Dhont 1999 J. Chem. Phys. 1107037

[41] M Kollmann and G Nägele 2000 J. Chem. Phys. 1137672

[42] J Rotne and S Prager 1969 J. Chem. Phys. 504831

[43] J M G Barthel, H Krienke and W Kunz 1998 Physical Chemistry of Electrolyte Solutions (Darmstadt: Springer)

[44] E C Zhong and H L Friedman 1987 J. Sol. Chem. 16337

[45] A Wilk, J Gapinski, A Patkowski and R Pecora 2004 J. Chem. Phys. 12110794

[46] G A Schumacher, T G M van de Ven 1991 J. Chem. Soc. Faraday Trans. 87971

[47] A Vizcarra-Rendon, M Medina-Noyola and R Klein 1990 Chem. Phys. Lett. 173397

[48] X Schlagberger, J Bayer, J O Rädler and R R Netz 2006 Europhys. Lett. 76346

[49] T Araki and H Tanaka 2008 Europhys. Lett. 8218004

[50] H Kodama, K Takeshita, T Araki and H Tanaka 2004 J. Phys.: Condens. Matter 16 L115

[51] K Kim, Y Nakayama and R Yamamoto 2006 Phys. Rev. Lett. 96208302

[52] Y Nakayama and R Yamamoto 2005 Phys. Rev. E 71036707

[53] V Lobaskin, B Dünweg, C Holm, M Medebach and T Palberg 2007 Phys. Rev. Lett. 98176105

[54] V Lobaskin, B Dünweg and C Holm 2004 J. Phys.: Condens. Matter 16 S4063 